$(<1-36)$, and 10 years working in palliative medicine ( $<1$ year -31 years, one working as bank).

Most reported confidence in recognising and managing TA as well as the specific use of midazolam, haloperidol and levomepromazine. Respondents were less familiar with phenobarbital (table).

Lowest levels of confidence in managing TA were reported in respondents with the least experience of this.

Free text replies indicated that making patients settled was the most rewarding aspect of managing TA; impact on family was another common theme.

Respondents however described a number of challenges including difficulty in recognising $\mathrm{TA}$, and regarding medication decisions.

Conclusions With support of a local guideline, high levels of confidence were reported in use of first and second line medications for TA. However respondents still reported challenges in its management.

Future considerations We recommend regular education and training alongside ongoing reflection amongst medical and nursing teams after events of TA, to ensure best practice, team well-being and confidence with guidelines.

\section{P-97 EXPLORATION INTO USE OF CONTINUOUS SUBCUTANEOUS LEVETIRACETAM WITHIN PALLIATIVE CARE}

${ }^{1}$ Matthew Dore, ${ }^{1}$ Clare Marlow, ${ }^{2}$ WM CARES, Sharon Twigg. ${ }^{1}$ The Royal Wolverhampton NHS trust, West Midlands, UK; ${ }^{2}$ West Midlands Collaborative Actioning Research in End-oflife and Supportive Care, West Midlands, UK

10.1136/bmjspcare-2017-00133.96

With advances in medicine and people living longer with chronic medical conditions new subsets of patients emerge. One such subset is a group of patients for which seizure control is paramount yet they are not actively dying and their oral and intravenous route of administration has become unavailable/inappropriate. Traditional treatment for seizure management has been subcutaneous (S/C) midazolam, however this often does not balance Quality of Life (QOL) favourably for this interim period, primarily due to associated drowsiness. There have been numerous case reports using continuous subcutaneous infusion (CSCI) levetiracetam as an alternative for this group of patients, as a consequence of the perceived more favourable side effect profile.

We have collated the research published to date which outlines the appropriate scenarios and limitations of levetiracetam $\mathrm{S} / \mathrm{C}$ route (either intermittent or $\mathrm{CSCI}$ ). We have outlined the side effects, dose regimens and practical issued regularly encountered.

We have sought expert neurological advice and explored the limitations of other anti-epileptics via the $\mathrm{S} / \mathrm{C}$ route and noted hopeful upcoming newer therapies such as Brivaracetam and Lacosamide.

We have made recommendations regarding starting doses, disease aetiology considerations and practical titration and conversion issues.

Within this area of rapidly progressing research, collating what has been studied so far and gaining support out-with specialist palliative care organises our thoughts and creates an overview on which we can base future research and develop guidelines to encourage consistent safe practice.

\section{P-98 PATIENT AND CAREGIVER EXPERIENCES OF DO NOT ATTEMPT CARDIOPULMONARY RESUSCITATION (DNACPR) CONVERSATIONS: AN INTEGRATIVE REVIEW OF THE LITERATURE}

${ }^{1}$ Emma Carduff, ' ${ }^{1}$ Jean Lugton, ${ }^{1}$ Juliet Spiller, ${ }^{3}$ Charlie Hall. ${ }^{1}$ Marie Curie Hospice, Edinburgh, UK: ${ }^{2}$ Marie Curie Hospice, Glasgow, Scotland, UK; ${ }^{3}$ NHS Fife, UK

10.1136/bmjspcare-2017-00133.97

Background Following recent changes in UK case law, DNACPR decisions must be discussed with patients unless that conversation will cause harm. CPR should not be given if it is an inappropriate treatment. DNACPR conversations are therefore happening more frequently; yet evidence to support staff in knowing how to undertake these conversations is scarce from the patient/caregiver perspective. This integrative literature review aims to identify patient and family experiences of DNACPR conversations.

Methods A search of multiple databases was performed for adult patients and caregivers, from all settings worldwide (2004-2014). Abstracts were reviewed for relevance and quality. First person, retrospective accounts of patient/carer experience of DNACPR conversations were gold standard. Relevant full texts were appraised and recurring themes analysed and tabulated.

Results The initial search identified 559 abstracts. Of these, 46 full texts were deemed relevant including 9 UK and 37 international papers. Only 22 papers were original research with patients or carers who had experienced these conversations. Themes revealed the importance of DNACPR discussions being conducted by someone trusted and the importance of family/carer involvement, as well as perceived concerns about burdening family members. Timing preferences for discussions was variable revealing difficulties in finding the 'right time' to discuss. Discussions held at home or the GP surgery are preferable to discussions during acute admissions to hospital.

Conclusions This review highlights potential discrepancies between patient and carer preferences for DNACPR conversations, and the reality of how these conversations are currently taking place. Public health approaches to tackle the societal taboo around death and dying may encourage people to think about and expect such conversations during their illness trajectories. Discussion of DNACPR decisions with the patient is now a legal requirement, but patient centred care remains paramount. More evidence is essential to bridge these expectations.

\section{P-99 THE USE OF AN ONCOLOGICAL PALLIATIVE DEPRESCRIBING GUIDELINE TO AID RATIONALISING MEDICATIONS IN PATIENTS IN THE LAST SIX MONTHS OF LIFE}

Anil Prabhu, Anna Sutherland, Victoria Bradley, Helen Pegrum. Florence Nightingale Hospice, Aylesbury, UK

\subsection{6/bmjspcare-2017-00133.98}

Background It is widely recognised that large numbers of patients remain on unnecessary medications after transition from a curative to palliative pathway. This is often the result of a lack of vigilance and confidence amongst healthcare professionals when rationalising medications ${ }^{[1]}$. This can negatively impact on quality of life, through 'pill burdens' and 
side effects, and also places avoidable financial strain on the health service. An Australian group published an 'OncPal deprescribing guideline' to assist clinicians in identifying potentially inappropriate medications (PIMs), which was used to help reduce polypharmacy in patients discharged from our hospice ${ }^{[2]}$.

Methods Retrospective case note review of patients with a prognosis of six months or less discharged from our inpatient unit during two 2 month periods, was performed. PIMs were identified using the OncPal guideline and then assessed for appropriateness independently by two hospice doctors. Following baseline data collection, the hospice used posters and multidisciplinary team meetings to encourage use of the OncPal guideline in an effort to raise awareness and eradicate futile medicines.

Results Baseline data $(n=19)$ revealed that there were 61 PIMs on admission, of which $19.7 \%$ were deemed truly inappropriate by both doctors. Of these, $75 \%$ were discontinued, resulting in 0.16 truly inappropriate medications per patient on discharge. Between the 2 reviewing doctors, there was discordance over the perceived appropriateness of $19.7 \%$ of admission medications. Post-intervention data $(n=9)$ showed $54.8 \%$ of admission PIMs were considered truly inappropriate by both doctors, of which $35.3 \%$ were ceased, resulting in 1.22 truly inappropriate PIMs on discharge. However, discordance had dropped to $6.5 \%$.

Conclusions These results highlight the difficulties in managing medications in palliative patients. Whilst the OncPal deprescribing guideline may help healthcare professionals to identify PIMs, more interventions are needed to empower doctors to appropriately stop these medications, to the benefit of patients and the healthcare sector as a whole.

\section{P-100 NEEDS IDENTIFICATION AND PLANNING FOR PALLIATIVE CARE IN CHILDREN WITH LIFE-LIMITING CONDITIONS}

Archana Soman. Norfolk and Norwich University Hospital NHSFT, Norwich, UK

\subsection{6/bmjspcare-2017-00133.99}

Introduction The need for pro-active planning and delivery of multidisciplinary (MDT) care to children with life-limiting conditions (LLC) is widely acknowledged, and 'standards frameworks' have been recommended by Together for Short Lives and others. Palliative and end-of-life care are important priorities of the United Kingdom government.

Methods We conducted a retrospective case-notes audit of 20 children (8 cancer and 12 non-cancer), aged 28 days to 16 years, who had died as a direct result of a LLC, against seven pre-agreed standards. Sudden deaths, neonatal deaths and deaths within a month of diagnosis were excluded.

Results

- A 'breaking-bad-news' meeting was documented in $95 \%$ (Oncology 100\%, non-oncology 91.6\%), but this focussed on the diagnosis, and discussion of prognoses was lacking in most.

- A MDT meeting was documented in 85\% (Oncology: 100\%, non-oncology: 75\%).

- A key worker and a lead professional were both identified in 45\% (Oncology: 87.5\%, non-oncology: 16.7\%; $\mathrm{p}=0.01$ ).

- MDT assessment of palliative care needs was documented in 60\% (Oncology: 100\%, non-oncology: 33.3\%; $p=0.015$ ).
- Symptom management plans were found in 65\% (Oncology: 100\%, non-oncology: 41.7\%; $\mathrm{p}=0.0225$ ).

- Family and psycho-social needs were documented in $75 \%$ (Oncology: 100\%, non-oncology: 58.3\%).

- End-of-life care plans were documented in full in $40 \%$ (Oncology: 75\%, non-oncology: 16.7\%; $\mathrm{p}=0.0325$ ).

Recommendations and conclusion Recognition of needs and planning for palliative care in children remains sub-optimal overall, especially so in children with non-cancer LLC. This audit has led to much introspection and an acknowledgement of the need for hearts-and-minds change in clinicians' approaches. We hope to influence commissioners to develop a robust children's palliative care service with a complex-care co-ordinator, rolling MDT meetings and dedicated paediatrician time. A sub-regional working group is mapping services to needs, in order to identify further gaps. A comprehensive care pathway that will incorporate palliative and end-of-life care plans has been written, with input from parent groups.

\section{P-101 SOLSTICE: SANCUSO ${ }^{\circledR}$ IN SUPPORTIVE AND PALLIATIVE CARE; A FEASIBILITY STUDY IN PATIENTS WITH CANCER AND REFRACTORY NAUSEA AND VOMITING}

${ }^{1}$ Emma Dean, ${ }^{2}$ Richard Berman, ${ }^{1}$ Shaun Villa. ${ }^{1}$ Experimental Cancer Medicine Team, The Christie NHS Foundation Trust, Manchester, UK; ${ }^{2}$ Palliative Care Support TeamThe Christie NHS Foundation Trust, Manchester, UK

\subsection{6/bmjspcare-2017-00133.100}

Background Nausea and vomiting ( $\mathrm{N}$ and $\mathrm{V}$ ) are common, debilitating symptoms in patients with cancer, often precipitating inpatient admission for subcutaneous/intravenous antiemetics and re-hydration. Currently, there are no evidence-based solutions and treatment algorithms differ across clinical practice. Some of these patients will experience difficulty swallowing tablets and/or are unable to keep oral medications down. Treatments for patients with cancer may also reduce the ability of the intestines to absorb medicines within a tablet.

SANCUSO $^{\circledast}$ (Granisetron Transdermal System [transdermal skin patch]) is indicated for the prevention of ( $\mathrm{N}$ and $\mathrm{V})$ in patients receiving moderately and/or highly emetogenic chemotherapy regimens. The SANCUSO ${ }^{\circledast}$ patch delivers consistent, predictable levels of granisetron throughout five days with smoother daily pharmacokinetics compared to daily dosing. The role of Sancuso in patients with cancer and refractory $\mathrm{N}$ and $\mathrm{V}$ which is unrelated to chemotherapy has not been explored.

Methods An open-label, randomised feasibility study comparing Sancuso with 'physician's choice' of antiemetic in patients with cancer and refractory $\mathrm{N}$ and $\mathrm{V}$. A feasibility study is required at this juncture as standard antiemetic treatment in this patient population is undefined, and the therapeutic efficacy of Sancuso requires appraisal before embarking on a larger randomised trial. To assess feasibility, objectives have been categorised into the four domains; (i) Recruitment assess the number of patients approached, consent rate, number of eligible patients and explore the methods used to identify potential patients (ii) Patients - willingness to participate and acceptability of the intervention (iii) Clinicians' - ability to recruit, which physician's choice is selected, experience including monitoring of prescribing practice in the control 